\title{
Hot cores and cold grains
}

\author{
C.M.WALMSLEY and P.SCHILKE \\ Max-Planck-Institut für Radioastronomie, Auf dem Hügel 69, D-5300 Bonn 1, \\ F.R. Germany
}

September 4, 1991

\begin{abstract}
A review is given of the evidence for depletion of heavy elements in dense molecular cloud clumps onto dust grain surfaces. Particular attention is given to the recent controversy over the nature of the dense clumps in NGC 2024. We also discuss recent ammonia observations of "hot core" regions and evidence that the $\mathrm{NH}_{3}$ abund ance is greatly enhanced.
\end{abstract}

Key words: dust mantles - molecules - abundances -depletions - hot cores

\section{Introduction}

In dense molecular cloud regions, a considerable fraction of the heavy element content of the material is in the form of ices frozen on dust grain surfaces. This is clear on the basis of the studies of solid state absorption features seen typically at near infrared wavelengths. Recent reviews of dust mantle composition and properties are by Tielens (1989) and by Whittet and Duley (1991). There is little doubt that deposition of heavy elements onto grain surfaces is of importance for the chemistry in high density clumps or cores within molecular clouds.

On the other hand, there is also little doubt that close to newly formed massive stars, dust temperatures can be sufficiently high that the greater part of such ice mantles are evaporated and their content is returned to the interstellar medium. The classic example is the "hot core" in Orion. Discussions of this region are given by Genzel and Stutzki (1989) and by Wilson and Walmsley (1989). It seems very plausible that what one is seeing in this region is essentially freshly evaporated grain mantle material. Models by Brown et al. (1988) and by Brown and Millar (1989) show that the observational data are basically consistent with this idea although there are a lot of open questions. A consequence is that one learns something about grain mantle composition by observing the Orion and other "hot cores".

In this short review, we give a brief summary of recent developments in this field. It could perhaps be subtitled "getting on and off grains". One would clearly like to find examples of regions where a large fraction of the heavy elements have been deposited onto dust grains and section 2 discusses some recent attempts to identify such regions. The other side of the coin is as mentioned above when one studies hot cores. Section 3 considers what is known about the properties of these regions with particular emphasis on the rather little that is known about sources other than Orion. Finally, section 4 gives a few tentative conclusions and suggestions for the future. 


\section{Evidence for freeze out in NGC 2024}

Evidently, if one believes that the gas seen in "hot core" regions is the product of evaporation of dust grain mantles, it follows that there are other regions where a large fraction of the heavy element content in the gas has condensed out onto dust grain surfaces. Identifying such regions has proven to be difficult. This is illustrated by several recent studies of the NGC 2024 molecular cloud where claims and counter-claims have been made concerning the fraction of molecules (and by inference heavy elements) in the dense gas surrounding the HII region NGC 2024 (Orion B) (Mezger et al. 1988, Schulz et al. 1991, Gaume et al. 1991, Mezger et al. 1991, Mauersberger et al. 1991). No completely clear picture has emerged from all these studies but a few points have been established.

As a preliminary, we discuss what one expects to observe in regions where a large fraction of heavy elements have condensed out onto dust grain surfaces . Here, we mean that a large fraction is condensed out relative to the "normal molecular cloud composition". Equivalently, the depletion factor $\delta$ of heavy elements (essentially $\mathrm{C}, \mathrm{N}, \mathrm{O}$ ) relative to solar abundances is larger than (say) 10-100. In molecular clouds, it is useful to use CO as a standard since $\mathrm{CO}$ is the sole observed (apart perhaps from $\mathrm{SiO}$ which is a special case) interstellar molecule to contain the greater part of the available gas phase supply of one of it's constituent elements (in this case carbon). Thus, the estimated $\left[\mathrm{CO} / \mathrm{H}_{2}\right]$ abundance ratio, which is typically $10^{-4}$ in normal low density molecular clouds might reasonably be taken as a measure of normalcy and evidence for a distinctly lower ratio (say below $10^{-5}$ ) as evidence that molecules have condensed out. Other molecules clearly cannot be expected to have abundances proportional to the degree of $\mathrm{C}, \mathrm{N}, \mathrm{O}$ depletion. In fact, a recent study by Pineau des Forêts et al. (1992) shows that at a density of $10^{4} \mathrm{~cm}^{-3}, \mathrm{NH}_{3}$ and $\mathrm{HCN}$ have abundances of around $10^{-8}$ relative to hydrogen independent of $\delta$ as long as $\delta$ is less than 1000. Such model studies may not represent reality in detail but they do demonstrate the dangers of using the abundances of trace species as proportional to the gas phase abundance of the heavy elements which they contain.

But let us suppose that there are high density clumps where the depletion factor is much higher even than 1000 and one can say that the heavy elements are frozen out. The most obvious observational criterion for freeze-out is a high ratio of the intensity of dust emission at, say, a wavelength of $1.3 \mathrm{~mm}$ to the intensity of some isotopic substitution of $\mathrm{CO}$ at a similiar wavelength. With this in mind, Mauersberger et al. (1991) have compared $\mathrm{C}^{18} \mathrm{O}(2-1)$ maps of NGC 2024 with the earlier $1.3 \mathrm{~mm}$ dust continuum maps of Mezger et al. (1988). One sees in both cases a ridge of material running roughly north-south with some "condensations" apparently superposed. The compact features are however much more clearly present in the dust emission 
map which additionally shows less extended material. Such differences may partly be due to the different dependence of dust and $\mathrm{C}^{18} \mathrm{O}$ radiation on temperature. However, Mauersberger et al. conclude that there is evidence in their data for $\mathrm{CO}$ depletion in the high density condensations seen in the dust map (where they estimate that the temperature is approximately $20 \mathrm{~K}$, the density $10^{8} \mathrm{~cm}^{-3}$, and the column density of order $10^{25} \mathrm{~cm}^{-2}$ ). Incidentally, the mass (of order 10 solar masses) and density of these clumps are similiar to those estimated for the Orion hot core. Thus, it is feasible that such condensations could eventually evolve into regions with parameters similiar to those of the hot core if a massive star were to form in their interiors.

The claim that there is evidence for molecular depletion is contested by Schulz et al. (1991) who present maps of CS(5-4) and CS(7-6) and compare them with the dust data. Perhaps more important than the differences between molecular line and dust emission maps are their similiarities. In the dense ridge gas (where $\mathrm{n}\left(\mathrm{H}_{2}\right)$ is of order $10^{6} \mathrm{~cm}^{-3}$ ), the distribution of molecular line emission and dust emission is very similiar. Differences make themselves apparent only around the embedded clumps. The CS and CO abundances in the general ridge gas appear to be normal and hence, one clearly has no great degree of depletion under these conditions. This itself is at first sight surprising given the expected freeze out timescale (roughly $10^{4}$ years for a sticking coefficient of 0.1 , see Walmsley 1991). The dynamical timescale for the region should be roughly $l / \Delta v$ where $l$ is size and $\Delta v$ is linewidth and thus it is also of order $10^{4}$ years. It is difficult to imagine that the NGC2024 ridge is younger than this and hence we conclude that there is reason to believe that the freeze-out time is being somewhat underestimated. The most obvious explanation of this is that, due to grain coagulation, the mean grain cross-section per hydrogen atom is less than in diffuse interstellar material. A relatively modest reduction in this parameter seems capable of explaining the present observational situation.

In the denser condensations mentioned earlier, the situation is much less clear. However, one important result is that VLA ammonia observations with $3^{\prime \prime}$ resolution detect clumps which in several cases are coincident with the dust emission clumps (Mauersberger et al. 1991). The apparent ammonia abundance is in the range $10^{-10}-10^{-9}$ depending on whether one uses the virial mass or the dust emission data to estimate the $\mathrm{H}_{2}$ column density. This is lower than "normal" but not drastically so and certainly the higher end of the range is within the uncertainties of current gas phase models. It is also significant that the temperatures inferred from the VLA $\mathrm{NH}_{3}$ observations are similiar to those $(20 \mathrm{~K})$ derived from the dust observations. This is much less than Schulz et al. obtain on the basis of their CS data $(35 \mathrm{~K})$. One explanation might be that these clumps are being heated from the outside and that consequently there are sharp temperature gradients from outside to 
inside. In this scenario, the VLA ammonia refers to an interior region with higher density but lower temperature than the gas seen in the single dish CS data. Obviously, it will be useful to obtain interferometric observations of species such as $\mathrm{CS}$ and $\mathrm{C}^{18} \mathrm{O}$ in order to verify this picture.

One should realise however that the observed ammonia abundance in the high density clumps (roughly $10^{-9}$ relative to $\mathrm{H}_{2}$ ) is compatible with the possibility that essentially all heavy elements have condensed out onto dust grain surfaces but that Lyman photons produced in these clumps as a secondary product of cosmic ray ionisation maintain a small fraction of molecules in the gas phase (see Hartquist and Williams 1990, Prasad and Tarafdar 1983 for discussions of this). In this situation, molecules such as $\mathrm{NH}_{3}$, which are relatively enhanced in abundance in dust grain mantles, may be as abundant as $\mathrm{CO}$. Following Hartquist and Williams, one finds that the number density $n\left(\mathrm{NH}_{3}\right)$ of ammonia molecules in such a steady state situation is given by :

$$
n\left(\mathrm{NH}_{3}\right)=0.075\left(\frac{\zeta_{H}}{10^{-17}}\right) S^{-1}\left(\frac{210^{-21}}{\overline{\sigma_{g}}}\right) f_{p h} f\left(\mathrm{NH}_{3}\right) \mathrm{cm}^{-3}
$$

Here, $\zeta_{H}$ is the hydrogen cosmic ray ionization rate and $S$ is the ammonia sticking coefficient. $f_{p h}$ is the quantum efficiency for release of mantle molecules per incident UV photon and $f\left(\mathrm{NH}_{3}\right)$ is the fraction of mantle molecules which are ammonia. Finally, $\overline{\sigma_{g}}$ is the mean grain cross section per hydrogen which in diffuse interstellar gas is estimated to be $210^{-21} \mathrm{~cm}^{2}$. The various efficiency factors in this equation are highly uncertain. However, one can certainly concoct plausible parameter combinations which are compatible with the observed VLA ammonia number density towards NGC2024 FIR3 which is roughly $0.01 \mathrm{~cm}^{-3}$ according to Mauersberger et al. (1991). Reasonable parameters for instance might be : $f\left(\mathrm{NH}_{3}\right)=0.03, f_{p h}=$ $0.1, S=0.1, \overline{\sigma_{g}}=210^{-22}$.

The best test of this hypothesis is to observe other molecules. Relative abundances should be similiar to those observed in the solid state (see e.g. Tielens) or in hot cores and quite different from normal molecular cloud material. In any case, one concludes paradoxically that gas phase molecules may be observable even when everything has frozen out. One needs to observe with an interferometer however in order to filter out normal gas!

\section{Hot core sources in the galaxy}

The Orion hot core is certainly not unique in the galaxy. Since one of its defining characteristics is a high ammonia column density in a region roughly $10^{17} \mathrm{~cm}$ in extent, one can use results from various recent ammonia surveys in order to isolate objects of similiar type. In table 1, we summarize data for an 
TABLE I

Parameters of galactic hot core sources

\begin{tabular}{cccccc} 
Source & Temperature & cDiameter & $\mathrm{N}\left(\mathrm{NH}_{3}\right)$ & $\log \left(\mathrm{N}\left(\mathrm{NH}_{3}\right) / \mathrm{N}\left(\mathrm{H}_{2}\right)\right)$ & \\
\hline Ori-KL & 160 & 0.025 & $1.310^{18}$ & -6.2 & HWWH \\
SgrB2-N & 200 & 0.2 & $1.010^{20}$ & & VGP \\
W31C & 100 & 0.026 & & -6.3 & MHWW \\
W51d & 250 & 0.06 & $2.010^{19}$ & -5.5 & MHWW \\
N7538 & 220 & 0.015 & $2.510^{18}$ & & MHWW \\
\hline
\end{tabular}

updated list of such sources. Data for table 1 has been taken from Hermsen et al. (1988,HWWH), Mauersberger et al. (1986,MHWW), and Vogel et al. (1987, VGP). The abundances given in table 1 should be treated with some scepticism. The main uncertainty is usually the molecular hydrogen column density in the "cores" and in several cases given in table 1, this has been estimated by assuming the virial theorem to hold in the clump and using the observed line width and size. This procedure is doubtful but not necessarily worse than the alternative of using measurements of the dust emission at miilimeter and sub-millimeter wavelengths. In this latter case, the problem is the highly uncertain dust emissivity at these wavelengths. One notes for example that Sievers et al. (1991) deduce a column density of $810^{24} \mathrm{~cm}^{-2}$ of $\mathrm{H}_{2}$ towards W51d from their $870 \mu \mathrm{m}$ measurements which compares with $610^{24}$ from the virial estimate. However, the latter result refers to a region roughly $0.06 \mathrm{pc}$. in diameter as compared to $0.25 \mathrm{pc}$. in the case of the dust emission measurements and hence there is effectively an order of magnitude difference between the two estimates.

Somewhat less uncertain but still to be treated with caution are the direct column density and temperature estimates based upon the molecular data. It is interesting in this context to compare the recent studies of NGC7538 by Keto(1991) and Schilke et al. (1991) with the earlier work by Mauersberger et al. (1986,MHWW). All three studies use partially independent data sets to derive the column density and rotation temperature of the hot ammonia clump seen towards NGC 7538-IRS1. The derived temperatures vary between $125 \mathrm{~K}$ (Keto, 1991), $150 \mathrm{~K}$ (Schilke et al. ), and $220 \mathrm{~K}$ (MHWW) whereas the $\mathrm{NH}_{3}$ column densities are consistent to within a factor 3 (Schilke et al. $10^{18.7}$, Keto $10^{18.2}$, MHWW $10^{18.4}$ ). These differences are probably due to density and temperature gradients within the source which have the consequence that results depend upon the particular set of transitions used for the analysis.

We have recently (Cesaroni and Walmsley 1992) made a small survey of galactic HII regions in the $(4,4)$ and $(5,5)$ transitions of ammonia with the aim of expanding the list in table 1 . This was based upon an earlier survey by Churchwell et al. (1990) where a large sample of galactic Hil regions were 
examined in $\mathrm{NH}_{3}(1,1)$ and $(2,2)$. Not too surprisingly, we find that there are generally small hot ammonia clumps associated with the compact HII regions. A surprise however was the observation of hyperfine satellites in the $(4,4)$ and $(5,5)$ lines towards G10.47+0.03 and G31.41+0.31. Simply interpreted, this implies very high line optical depths $(\tau(4,4) \sim 50-100)$ and correspondingly high ammonia column densities (greater than $10^{19} \mathrm{~cm}^{-2}$ ). The situation is thus rather similiar to that found by MHWW towards W51d. The size of the emitting region must be of the order of $1-2^{\prime \prime}$ or $10^{17} \mathrm{~cm}$ and thus we presume that the essential characteristics are similiar to those of the Orion hot core. The ammonia abundance must be extremely high and our best guess is around $10^{-5}$. One should stress that, as usual, this is very uncertain and the main uncertainty is the hydrogen column density. Another interesting point is that in a separate study, torsionally excited methanol was found in these two sources (Cesaroni et al.1991). In view of the probability that methanol is abundant on grain surfaces (see e.g. Grim et al. 1991), this seems to substantiate the general hypothesis that the abundance distribution in "hot cores" reflects that found in grain mantles and that the molecules observed in hot cores have recently been evaporated from mantles.

An obvious extension of this single dish work is to observe $\mathrm{NH}_{3}$ with an interferometer. Five of the $\mathrm{NH}_{3}(4,4)$ sources found by Cesaroni and Walmsley (1992) have recently been observed with the VLA (Kurtz and Churchwell, priv.comm.). Results from this study are very preliminary but already show that the sources of $\mathrm{NH}_{3}(4,4)$ emission are in general very compact and, in some cases at least, are emitted from a small clump very close to the compact HII region. The true ammonia brightness temperatures are clearly quite high (at least $40 \mathrm{~K}$ towards $\mathrm{G} 10.47+0.03$ ) which is important because it restricts the possibility that the ammonia transitions are in some way being cooled much below the kinetic temperature and that the high optical depths are partially a consequence of this "super-cooling".

\section{Conclusions}

The data to date support the contention that one requires high densities (above $10^{6} \mathrm{~cm}^{-3}$ ) before drastic depletion sets in. This does not prevent considerable depletion and indeed chemistry on grain surfaces at lower densities. At higher densities, one needs observations with arc second angular resolution in order to avoid confusion. The new millimeter interferometers which are becoming available should be a considerable help in this respect. This is true whether one is examining "hot cores" or clumps such as found in NGC 2024.

It is worth pointing out also that if one wants to distinguish effects of gas phase and dust chemistry, one needs information about the abundances of many species. Even in the Orion hot core, the present state of our knowl- 
edge is very vague due to confusion between the well known "zoo" of velocity components in and around Orion-KL. For example, there is still no reliable estimate for the $\mathrm{HCO}^{+}$abundance in the hot core. To resolve this and analogous problems, it would be useful to make a spectral line sweep with a sensitive millimeter interferometer. Thus, fortunately, there are problems left for the future.

\section{References}

Brown P.D., Charnley S.B., Millar T.J. 1988 Monthly Notices Roy. Astron. Soc. , 231,409. Brown P.D., Millar T.J. 1989 Monthly Notices Roy. Astron. Soc. , 237,661.

Cesaroni R., Walmsley C.M., Churchwell E. 1991 Astron. Astrophys. (in press).

Cesaroni R., Walmsley C.M. 1992 Astron. Astrophys. (in press).

Churchwell E., Walmsley C.M., Cesaroni R. 1990 Astron. Astrophys. Suppl. ,83,119.

Gaume R., Johnston K.J., Wilson T.L. 1991 Astrophys. J. (in press).

Genzel R., Stutzki J. 1989 Ann. Rev. Astron. Astrophys. 27,41.

Grim R.J.A., Baas F., Geballe T.R., Greenberg J.M., Schutte W. 1991 Astron. Astrophys. 243,473

Hartquist T.W., Williams D.A. 1990 Monthly Notices Roy. Astron. Soc. , 247,343.

Hermsen W., Wilson T.L., Walmsley C.M., Henkel C. 1988 Astron. Astrophys. 201,285.

Mauersberger R., Henkel C., Wilson T.L., Walmsley C.M. 1986 Astron. Astrophys. $162,199$.

Mauersberger R., Wilson T.L., Mezger P.G., Gaume R., Johnston K.J. 1991 Astron. Astrophys. (in press).

Mezger P.G., Chini R., Kreysa E., Wink J.E., Salter C.J. 1988 Astron. Astrophys. 191,44.

Mezger P.G., Sievers A.W., Haslam C.G.T., Kreysa E., Lemke R., Mauersberger R., Wilson T.L. 1991 Astron. Astrophys. (in press).

Pineau des Forêts G., Flower D.R., Millar T.J. 1992 Monthly Notices Roy. Astron. Soc. (submitted)

Prasad S.S., Tarafdar S.P. 1983 Astrophys. J. , 267,603.

Schilke P., Walmsley C.M., Mauersberger R. 1991 Astron. Astrophys. 247,487.

Schulz A., Güsten R., Zylka R., Serabyn E. 1991 Astron. Astrophys. 246,570.

Sievers A.W., Mezger P.G., Gordon M.A., Kreysa E., Haslam C.G.T., Lemke R. 1991 Astron. Astrophys. (in press)

Tielens A. 1989 p239 in Interstellar Dust, Proceedings of IAU Symposium 135, (ed. Allamandola L.J., Tielens A.G.G.M. ; publ. Kluwer).

Vogel S.N., Genzel R., Palmer P. 1987 Astrophys. J. , 316,243.

Walmsley C.M. 1991 in Fragmentation of Molccular Clouds and Star Formation, IAU Symposium 147, (ed. E.Falgarone et al.)

Whittet D.C.B., Duley W.W. 1991 The Astron. and Astrophys. Review, 2,167.

Wilson T.L., Walmsley C.M. 1989 The Astron. and Astrophys. Revievo, 1,141. 


\section{QUESTIONS AND ANSWERS}

A.Dalgarno: To oversimplify, if there is no depletion, as you concluded, how can there be a grain chemistry? C.M.Walmsley: When I said "no depletion", I meant "no drastic depletion" (i.e. loss of a major fraction of CNO) in the NGC 2024 ridge. There certainly is some depletion as evidenced by solid state features seen towards IR sources.

M.Ohishi: We have observed vibrationally excited $\mathrm{CH}_{3} \mathrm{OH}$ up to $v_{t}=2(E \sim 700 \mathrm{~K})$ in many active star forming regions (M.Ohishi et al. in prep.). Our results show $T_{\text {rot }}$ in one vibrational state and $T_{\mathrm{r} i b}$ among vibrationally state are very similar, and we think vibrationally excited $\mathrm{CH}_{3} \mathrm{OH}$ is mainly excited by collision. But you mentioned that vib. excited $\mathrm{CH}_{8} \mathrm{OH}$ traces FIR. I would like to know the reason.

C.M. Walmsley: Probably both collisions and radiation play a role. The collisional rates between torsional states in $\mathrm{CH}_{3} \mathrm{OH}$ is quite unknown as far as I can tell and the relative importance of collisions and radiation depends on this.

A.Leger: What is the core life time so that we can compare it to the accretion time on grains?

C.M.Walmsley: The cores have a "dynamical lifetime" defined by size and linewidth which is $\sim 3 \times 10^{4}$ yrs. This is probably a lower limit.

N.Evans: There certainly is a way to get some of the molecules off the grains. There are several hundred young stars in the NGC 2024 region and these will heat grain mantles in at least the outer parts of the clumps.

C.M.Walmsley: The extinction in the ridge is $\sim 100 \mathrm{mag}$ and the radiation from the stars has to penetrate that.

J.M.Greenberg: $T_{d}$ is uncoupled from $T_{g}$ for $n_{H}<10^{\alpha} \alpha>10$. I see no problem in having $T_{d}<T_{g}$ at the gas densities you are talking about. A simple way of looking at this (which I will confirm in detail later) is that at $16 \mathrm{~K}$ the dust is radiating energy at a rate $>0.1 \mathrm{eV}$ (energy density) $\times 3 ; \times 10^{10}$ (velocity) $=$ $3 \times 10^{\circ}$ and if gas is at $30 \mathrm{~K}$ it can impart energy to the dust at a rate $\sim 0.003 \mathrm{eV}$ (energy per $\mathrm{H}$ ) $\times n_{H} \times$ $v_{H} \simeq 3 \times 10^{-8} n_{H} 10^{4} \mathrm{~cm} / \mathrm{s} \geq$ radiation rate if $n_{H}>10^{8} \mathrm{~cm}^{-8}$.

C.M.Walmsley: I think the dust will cool the gas rather than the gas heating the dust. The question is what is heating the gas. 